821.131.1.09-84

https://doi.org/10.18485/italbg.2017.1.3

\author{
Francesca Pucci Donati* \\ Università di Bologna
}

\title{
OSTI E OSTERIE NEI PROVERBI ITALIANI FRA MEDIOEVO ED ETÀ MODERNA
}

\begin{abstract}
L'ospitalità a pagamento ha sempre costituito un tema ricorrente nella letteratura in volgare. La taverna, l'osteria e l'albergo, infatti, sono spesso menzionati nei detti medievali al pari del taverniere, dell'oste e dell'albergatore. Questi professionisti sono frequentemente raffigurati quali simboli di furbizia e avidità, raramente di onestà e generosità. Sono spesso individui che mercificano i rapporti umani e ingannano gli altri. La donna, in particolare, è di solito descritta come una meretrice dedita alla vita dissoluta. L'uomo, invece, non è soltanto rappresentato come un mercante attento ai propri affari; può anche incarnare l'oste che svolge il proprio lavoro onestamente. I proverbi relativi all'ospitalità, perciò, hanno sempre un significato etico che trascende il mondo dell'osteria stessa. Essi stigmatizzano certi comportamenti e qualità negative dell'uomo, ma nel contempo contribuiscono a creare nell'immaginario popolare un'idea dell'oste, che è rimasta viva fino a oggi. In tal senso, la cultura proverbiale fornisce diverse chiavi di lettura della società, delle credenze e dei costumi del passato.

Parole chiave: ospitalità, proverbi, Medioevo, osti, taverna, moralità.
\end{abstract}

Tre cose solamente mi so' in grado, le quali posso non ben ben fornire: ciò è la donna, la taverna e 'l dado; queste mi fanno 'l cuor lieto sentire.

Così si esprime il poeta senese Cecco Angiolieri (1990: 146) nel celebre sonetto LXXIV delle sue Rime. Numerosi sono gli esempi attestati nella letteratura in lingua volgare, sin dalle origini, nei quali la taverna è associata al piacere e al divertimento e, del pari, alla vita dissoluta e al malaffare. Basti pensare a diversi componimenti duecenteschi, come per esempio i Proverbia super natura feminarum di un anonimo settentrionale,

\footnotetext{
*francesca.pucci@unibo.it
} 
che attestano l'espressione "Lo gloto a la taverna molto ne va corendo; / la donna tavernara recevelo ridendo" (Contini 1960: 544) ${ }^{1}$; oppure alla riflessione di Brunetto Latini (1985: 98), che scrive nel Tesoretto: "E tegno grande scherna / chi dispende in taverna ; / e chi in ghiottornia / si getta, o in beveria, / è peggio che omo morto / e 'l suo distrugge a torto". Ancora, nel XIV secolo Paolo da Certaldo (1945: 137) nel suo Libro di buoni costumi ricorda l'ammonimento dantesco "si dee usare in taverna co' ghiottoni, e in chiesa co' santi, e in inferno co' dimoni". In testi di questo tipo la taverna e l'osteria sono evocate come simbolo di un microcosmo dove una molteplicità di attività anche ludiche hanno luogo intorno al mangiare, al bere e alla convivialità. Si tratta, in parte, di una letteratura contraddistinta da una vena comica, come le rime di Cecco Angiolieri, in cui la dimensione della taverna compare con un'accezione positiva. In diverso genere di letteratura, ed è più spesso il caso della poesia didattica e della novellistica, la taverna risulta invece essere connotata quale luogo equivoco, malfamato, dove si incontrano soprattutto meretrici e truffatori. In tal senso, il mondo dell'ospitalità è descritto per esempio nelle novelle XXXIII e CLXXXVI del Trecentonovelle di Franco Sacchetti (2004: 139, 525), dove la taverna è citata in due proverbi posti alla fine delle novelle a mo' di motto conclusivo. Si tratta nella fattispecie del proverbio "Una pensa el ghiocto e l'altra el tavernaio", impiegato dall'autore in veste di avvertimento nei confronti di coloro che si sentono più furbi degli altri, ma non si accorgono che potrebbero imbattersi in qualcuno ancora più scaltro. Il tavernaio, in particolare, è paragonato al ghiottone: se quest'ultimo escogita qualsiasi stratagemma per trovare il proprio tornaconto nel mangiare a spese di terzi, l'oste, d'altro canto, non si lascia cogliere impreparato e riesce a muovere tutte le situazioni a proprio favore.

La taverna e l'albergo, al pari dell'oste, dell'albergatore e del taverniere, ossia i luoghi e i mestieri dell'ospitalità a pagamento nel Medioevo, vengono ad arricchire uno dei soggetti ricorrenti nella letteratura proverbiale in volgare a partire dal XIII secolo. Con letteratura proverbiale intendiamo l'insieme vastissimo di formule in lingua italiana inserite nei testi degli autori (di qualsiasi genere) e, ugualmente, quelle inserite nelle liste di proverbi, definite comunemente dai filologi 'serie alfabetiche proverbiali' ${ }^{2}$. Questo secondo genere di micro-testi è stato spesso chiamato 'testo senza contesto', in riferimento alla mancanza di un contesto culturale in cui verosimil-

${ }^{1}$ La localizzazione veneta dei Proverbia super natura feminarum individuata da Contini è stata recentemente messa in discussione. Al riguardo, Pier Vincenzo Mengaldo ha proposto in alternativa una localizzazione del testo di ambito cremonese (Mengaldo 2012: 19-35).

${ }^{2}$ Riguardo alle serie proverbiali medievali vedi Novati (1910). 
mente poter inserire il proverbio ${ }^{3}$. Ciò non toglie che si tratta pur sempre di espressioni diffuse nella cultura scritta del tempo, in quanto ritroviamo buona parte degli stessi proverbi citati in opere di vario tipo, segno che si stava costituendo una sorta di repertorio collettivo, scritto oltre che orale, conosciuto da coloro che per mestiere redigevano testi, non necessariamente letterari. Anche se ritorna difficile ricostruire per la maggior parte delle espressioni la prima citazione e il primo contesto di produzione, tuttavia il confronto incrociato con altre fonti letterarie, ma anche scientifiche, come per esempio, i trattati di botanica o di medicina, o ancora, i manuali di buone maniere e i manuali di grammatica, permette di ricomporre un contesto d'uso dell'espressione e talvolta consente di valutare il grado di ricezione del proverbio. Nondimeno, come è stato già ampiamente notato in ambito letterario, linguistico e filologico, il proverbio può esprimere una consuetudine, una credenza, o comunque un'idea intorno a un tema, comunemente condivise dai più. In tal senso, esso ha un'importante valenza culturale e fornisce elementi di riflessione circa l'ambiente sociale ed economico cittadino in cui viene prodotto 4 .

Molteplici sono i temi che ricorrono nei proverbi sin dall'antichità; alcuni risultano essere rappresentati più di altri, soprattutto quando l'argomento stesso viene percepito da chi scrive e da chi legge o da chi l'intende pronunciato, come un esempio educativo e moralizzante. È certamente il caso dell'ospitalità a pagamento, un tema che si presta particolarmente a suggerire ed evocare giudizi etici sui comportamenti, sulle attività e sui costumi di vita. Del pari, i cibi e le bevande della taverna offrono all'immaginario collettivo degli efficaci simboli della convivialità, della socievolezza, ma anche dei rapporti di forza e di violenza esistenti fra le diverse categorie sociali che affollavano nel Medioevo il variegato mondo delle locande. Le figure degli osti e dei tavernieri, per esempio, sono spesso menzionate nei proverbi quali tipizzazioni umane e sociali, al fine di stigmatizzare determinati comportamenti e valori, come per esempio l'idea del tornaconto personale e del profitto. Similmente, le meretrici sono identificate con persone di dubbia moralità, unicamente mosse dall'interesse personale. La presenza nelle formule proverbiali di oggetti evocatori del gioco, quali i dadi e le carte, richiama inoltre l'immagine del giocatore, sempre rappresentato come un truffatore e approfittatore degli altri. L'universo della locanda, dell'albergo e della taverna, dunque, risulta essere caratterizzato nella cultura proverbiale da personaggi ambigui, eticamente riprovevoli e da disprezzare. Le variegate attività che si svolgevano nelle locande sono

\footnotetext{
${ }^{3}$ Vedi sull'argomento Shapin (2001). Cfr. Montanari (2008: 121-123).

${ }^{4}$ Circa l'importanza del proverbio nella cultura medievale e la rilevanza del contesto in cui esso viene prodotto, vedi Schulze-Busacker (2012).
} 
menzionate nei proverbi soprattutto per suscitare nel lettore o nell'ascoltatore un sentimento di rimprovero, meno spesso un senso di piacere e di divertimento. Il giudizio morale è sempre nascosto dietro questo genere di espressioni, anche laddove venga espresso un parere positivo nei confronti del lavoro dell'oste.

Il mestiere di quest'ultimo è oggetto in effetti di un'attenzione particolare nei proverbi, in quanto l'ospitalità a pagamento costituiva nel pieno e nel tardo Medioevo un servizio pubblico importante nelle grandi città europee, ma anche in quelle di media e piccola estensione ${ }^{5}$. Infatti, nell'Europa medievale l'oste, l'albergatore e il taverniere (risulta difficile distinguere il significato di tali termini) gestivano strutture a pagamento (o ne erano i proprietari), comprensive di vitto e di alloggio, per uomini e animali a seguito. Queste strutture si componevano normalmente di vari ambienti: accanto alla locanda vera e propria vi erano sempre le stalle per gli animali degli avventori e i locali che fungevano da magazzini delle merci per i mercanti viaggiatori che vi sostavano o alloggiavano per qualche giorno. La storiografia italiana $^{6}$ ed europea ${ }^{7}$ ha portato molti esempi in proposito: le osterie nel tardo Medioevo erano luoghi di convivialità, di socialità, di contrattazioni, ma anche di aspri scontri sociali. In esse si mangiava assieme, si discuteva, si stipulavano contratti, fra cui quelli di matrimonio, di lavoro, di eredità, etc.; si dirimevano le controversie; si giocava alle carte e a ai dadi; si assisteva a baruffe e litigi di ogni sorta. Le osterie erano anche dei piccoli mercati coperti, in quanto gli osti e i tavernieri vendevano i prodotti ai clienti che non li consumavano sul posto, ma li acquistavano per portarli via: grano, vino, pane, pesce, carne, talora piatti già pronti. Nei centri minori le taverne dovevano rimpiazzare il mercato giornaliero o settimanale, svolgendo un servizio importante per l'intera comunità. In questo senso il taverniere era un mercante al dettaglio dei beni alimentari di prima necessità e di quelli di largo consumo, che comprava sul mercato urbano per rivenderli nella locanda. Il taverniere, l'oste e l'albergatore, inoltre, rivestivano spesso il ruolo di funzionari pubblici, in quanto riscuotevano la tassa sull'ingresso in città delle mercanzie destinate alla vendita, trasportate dai forestieri che alloggiavano presso di loro. Essi riscuotevano, inoltre, il dazio sulla vendita del vino e su altri prodotti; ma potevano anche esigere la tassa sulle merci

${ }^{5}$ Una sintesi sui mestieri dell'ospitalità nel Medioevo è fornita in Peyer (1990). Cfr. Mazzi (1997: 113-129) e Gensini (2000).

${ }^{6}$ Alcuni studi in questo senso sono per esempio quelli di Tuliani (1994), Cherubini (1996, 1997), Tagliabue (2003), Balletto (2010, 2014).

${ }^{7}$ Vedi Gautier (2012); Freedman (2012); Kümin (2012). 
collocate presso i locali dell'osteria che fungevano da magazzini ${ }^{8}$. Accadeva spesso, infatti, che i mercanti vi depositassero le loro mercanzie, in attesa di smerciarle nei giorni di mercato.

Il mondo delle osterie, come detto, è dunque finemente e argutamente descritto, seppur in maniera impressionistica, nella letteratura proverbiale in lingua volgare, a partire dal tardo Medioevo sino ai giorni nostri, nelle opere degli autori e nelle serie alfabetiche proverbiali, comunemente definite dal XVI secolo in avanti, repertori e dizionari di proverbi ${ }^{9}$. Diverse sono le immagini che tale letteratura ci restituisce in proposito; tutte, nondimeno, concorrono a fornire un'idea della locanda tale e quale essa risulta dalle fonti documentarie e da quelle iconografiche coeve. Emerge forte, per esempio, nella letteratura proverbiale, la dimensione di mercato e degli affari che si svolgevano all'osteria e, per estensione di significato, quella della monetarizzazione dei rapporti umani. Nel detto medievale "Strettezza di signor, putane et hosti / essere non può già mai che non ti costi" (Pucci Donati 2012: 80), per esempio, l'oste, paragonato al signore e alla meretrice, figura come colui che è interessato e attento unicamente al proprio guadagno. Al pari della scarsa generosità del signore (anch'essa, è una delle tipizzazioni ricorrenti nei proverbi), qualsiasi genere di servizio a pagamento costa caro. L'evocazione del supposto consistente guadagno del gestore e, per contraltare, dell'elevato costo dei servizi a carico della clientela, costituisce un Leitmotiv nei detti dedicati al mondo delle locande. Come si può notare dall'esempio appena riportato, anche la figura femminile assume spesso una posizione di rilievo nella struttura sintattica dei proverbi. La donna, identificata esclusivamente con la meretrice, viene sempre associata a costumi discinti e dissoluti.

Il paragone fra il mondo dell'osteria e quello della prostituzione è in effetti ricorrente in molti proverbi medievali, come è il caso, per esempio, di "Ni in putana vegia ni in taverna nova no te fidare" (Pucci Donati 2012: 79). Occorre non fidarsi né della meretrice vecchia né della taverna che è stata aperta da poco, in quanto difficilmente potrà fornire gli stessi servizi di quella che ha una fama consolidata e una clientela abituale. Il tema della fiducia negli altri, e per contrasto, quello della diffidenza, sono per lo più associati alla taverna che viene spesso descritta quale luogo della mercificazione dei rapporti umani. Così pare evincersi dal detto "Taverna fa putta / femmina ghiotta" registrato da Garzo dell'Incisa nei suoi Proverbi (Brambilla Ageno 1984: 32), come pure dall'affine "Taverna fa putta / fem-

${ }^{8}$ Circa l'importanza dei luoghi dell'ospitalità nel sistema fiscale delle città medievali, vedi Rinaldi (2016: 170).

${ }^{9}$ Il repertorio di proverbi medievali proposto qui di seguito nel testo è ripreso da Pucci Donati (2012: 70-81). 
mina occhiutta", attestato in una delle diverse serie alfabetiche proverbiali medievali (Pucci Donati 2012: 81). La taverna trasforma qualsiasi genere di donna, dalla golosa ("ghiotta") alla scaltra ("occhiutta"), in meretrice pronta ad approfittare dell'occasione del momento e truffare il cliente sprovveduto. Si tratta di proverbi che rientrano nell'ambito di una letteratura di stampo misogino, molto diffusa nell'Occidente medievale, di cui lo Splanamento de li Proverbi de Salomone di Girardo Patecchio da Cremona (Contini 1960: 560-583) e i già citati Proverbia super natura feminarum di un anonimo settentrionale (Contini 1960: 523-555) costituiscono un chiaro ed evidente esempio. In questa letteratura, la donna è apostrofata esclusivamente come persona di cattivi costumi e dedita al malaffare; la sua immagine è perciò costantemente connotata in maniera negativa. La rappresentazione delle "tipizzazioni" umane, contraddistinte da un'etichetta morale, è del resto, come già sottolineato, una delle caratteristiche principali della letteratura proverbiale medievale. Il proverbio, grazie alla sua brevità e concisione, era ritenuto efficace dagli intellettuali e dai chierici del tempo (ma non soltanto da costoro), per trasmettere messaggi chiari e forti a un pubblico variegato, sensibile alla cultura scritta, e più spesso immerso totalmente nel mondo dell'oralità. Così, i predicatori parlavano ai credenti anche per proverbi; nelle scuole si insegnavano le regole del latino mediante l'uso di proverbi; i legislatori ugualmente si avvalevano di proverbi per redigere le leggi; i manuali di buon costume erano infarciti di regole a mo' di massima, che venivano impartite ai figli delle famiglie borghesi nel corso della loro educazione ${ }^{10}$. Le formule brevi, dunque, erano impiegate nel vivere concreto delle comunità quali norme pratiche da seguire e facevano parte di una memoria collettiva affidata alla cultura orale.

L'uomo, al pari della donna, si qualifica nei proverbi sull'ospitalità prevalentemente mediante il mestiere che svolge; in misura minore per la posizione sociale che ricopre, come è il caso più sopra citato della "strettezza del signore". Se però la donna esercita un'attività condannata moralmente già in partenza, l'oste, il taverniere e l'albergatore possono invece suscitare rispetto, se agiscono correttamente e nella tutela dell'avventore; o, diversamente, suscitare riprovazione e sdegno, se volti soprattutto al guadagno. Il giudizio è basato esclusivamente sul valore etico di colui che lavora nella locanda e su come esercita la propria attività. In questo senso anche l'aspetto esteriore dei luoghi ha una certa importanza e rispecchia la qualità del servizio offerto nella taverna, come recitano i due seguenti proverbi : "Buono albergo e mal oste" e "Buono oste e mal albergo" (Pucci Donati 2012: 75). Essi sembrano invitare il viaggiatore a scegliere con accortezza dove alloggiare: non conviene lasciarsi lusingare da locande apparentemente piacevoli

\footnotetext{
${ }^{10}$ Zemon Davis (1980: 309-361), Bautier (1984); Buridant (1984).
} 
("Buono albergo"), ma gestite malamente e in maniera disattenta dall'oste ("mal oste"). L'esperienza insegna, ed è il secondo dei due detti citati, che è invece preferibile sostare presso osterie apparentemente modeste, disadorne o scarsamente confortevoli ("mal albergo"), eppure corredate da un servizio puntuale ed efficace ("Buono oste"). Al pari dell'espressione precedente, ma in termini opposti, l'aggettivo qualitativo fornisce una valutazione etica del lavoro: "buono" è l'oste che si prende cura delle necessità e dei bisogni dei clienti. L'impiego delle coppie di espressioni associate in maniera oppositiva in entrambe le formule sottolinea la contrapposizione fra coloro che svolgevano il mestiere di oste intendendolo come un servizio pubblico da fornire ai viaggiatori, e coloro che invece lo consideravano unicamente quale attività di lucro e profitto. Le insegne dovevano ricoprire un ruolo importante in tal senso: disposte in maniera ben visibile, esse avvertivano $i$ passanti che in quel luogo si forniva un servizio pubblico a pagamento. La legislazione di numerose città europee medievali è eloquente in proposito; diverse norme, infatti, erano rivolte ai gestori e proprietari delle locande, tenuti per l'appunto a munire la loro struttura esternamente di insegne.

L'iconografia popolare di età moderna (XVI-XVIII secolo) fornisce esempi che suffragano questa ipotesi. Per esempio, dalla stampa Gioco di tutte le insegne delle osterie di Giuseppe Maria Mitelli ${ }^{11}$, pittore e incisore bolognese vissuto fra la fine del Seicento e l'inizio del Settecento, si delinea chiaramente, seppur in forma giocosa e scherzosa, il significato che l'insegna rivestiva nell'immaginario popolare. Per ogni riquadro, ciascuno consacrato a un'osteria specifica della città di Bologna, sono indicati: nome dell'osteria, via in cui essa era situata, specialità gastronomica per cui l'osteria era rinomata, oltre ovviamente al simbolo dell'osteria stessa. Così, la configurazione esteriore della locanda metteva immediatamente l'avventore nelle condizioni di poter fare una scelta in base ai propri gusti e alle proprie necessità. L'informazione sulle specialità gastronomiche rientrava in una logica di trasparenza e di onestà nei confronti degli avventori, che doveva esistere ben prima di Mitelli e del suo Gioco. Anche se simili testimonianze iconografiche non si riscontrano nel XV secolo, tuttavia è già medievale, come si è detto, l'idea che l'ospitalità a pagamento dovesse offrire strutture e servizi di accoglienza a supporto delle autorità governative nei centri cittadini, nei villaggi e nelle campagne.

La letteratura proverbiale dell'inizio dell'età moderna riproduce sostanzialmente i detti medievali, talvolta in maniera identica, talaltra riportando delle varianti, dovute alle rivisitazioni da una lingua volgare a un'altra (molte affinità si riscontrano per esempio fra i proverbi italiani, francesi e spagnoli), oppure derivanti dalle traduzioni dalle lingue antiche a quelle vernacolari.

\footnotetext{
${ }^{11}$ Mitelli (1988).
} 
Si riscontrano inoltre negli autori del Cinque-Seicento e nei repertori coevi, nuove espressioni, frutto della cultura del tempo, che in parte confluiranno nelle raccolte successive fino a essere registrate nei dizionari ottocenteschi. D'obbligo, per il caso italiano, è il riferimento ai Proverbi toscani del poeta e letterato Giuseppe Giusti, dizionario pubblicato postumo nel $1853^{12}$, divenuto un classico alla fine dell'Ottocento e riconosciuto tale ancora oggi. Diversi sarebbero i testi che si potrebbero citare al riguardo. Volendo in questa sede proporre soltanto qualche riflessione a margine di un discorso sui proverbi e sul mondo dell'ospitalità, ci limiteremo a un campione di esempi tratti dall'opera di uno scrittore cinquecentesco, che bene si presta a illustrare la circolarità fra cultura scritta e cultura orale nel Rinascimento italiano. Si tratta, nella fattispecie, di Giulio Cesare Croce, cantastorie bolognese, vissuto nella seconda metà del Cinquecento e ai primi del Seicento, autore di componimenti in volgare e in dialetto, nonché di tre repertori di proverbi e di formule brevi ${ }^{13}$. In realtà Croce non aveva realizzato tali repertori in funzione di una loro pubblicazione, quanto piuttosto sotto forma di brogliacci a uso personale, da cui ricavare delle espressioni da inserire nelle opere narrative. In tal senso, essi risultano ancora più interessanti in quanto l'autore li aveva raccolti e annotati perché probabilmente conosciuti e diffusi nella cultura scritta e orale, e perciò recepiti da un pubblico ampio e variegato.

Dall'esame dei testi di Croce, emerge che il tema del cibo viene privilegiato rispetto ad altri ambiti del vivere umano ${ }^{14}$. I mestieri dell'alimentazione, inoltre, e in special modo quelli dell'ospitalità, ricevono dal cantastorie bolognese un'attenzione particolare, data la centralità del mondo della taverna nella cultura popolare. Si tratta di un universo declinato quasi esclusivamente al maschile. Uomini sono per esempio i gestori dei locali e i loro dipendenti, come è il caso degli apprendisti garzoni: "Huomo scaltrito, saria buon garzone per un hoste" (Montanari \& Pucci 2009: 172). L'incipit del proverbio ("Huomo") evoca il tipo allegorico dell'uomo comune, già diffuso nel Medio Evo e protagonista anche nei proverbi di età moderna. Ritorna in questo detto una delle qualità ritenute vitali per un oste e per coloro che lavorano accanto a lui, ossia la scaltrezza. Soltanto chi è scaltro e avveduto può divenire un buon aiutante dell'oste. Quest'ultimo doveva essere pronto a scoprire e a rintuzzare qualsiasi tipo di truffa o di raggiro tramato dall'avventore che, dal canto suo, cercava sempre di far tornare i conti a proprio favore. Non a caso, gli osti sono sovente presentati quali

${ }^{12}$ Giusti (1993).

${ }^{13}$ Montanari \& Pucci (2009).

${ }^{14}$ I proverbi di Giulio Croce citati di seguito nell'articolo sono tratti dal repertorio contenuto in Montanari \& Pucci (2009: 165-175). 
antagonisti dei loro clienti, come si rileva nel già citato "Una cosa pensa il ghiotto, l'altra il tavernaro" (Montanari \& Pucci 2009: 175). Si tratta di un proverbio di cui si possono cogliere le tracce nei testi medievali, in quelli cinquecenteschi e successivi, fino ad arrivare ai Proverbi toscani di Giusti e oltre. È una delle tante testimonianze di come alcuni cosiddetti 'luoghi comuni' relativi alla taverna sono rimasti vivi nella cultura proverbiale e nell'immaginario collettivo sino a oggi.

La figura dell'oste, d'altronde, può avere un'accezione positiva, qualora diventi dispensatore di benessere per gli avventori, svolgendo con cura il proprio lavoro. Così Croce annota i detti "Huomo amorevole, saria buon oste" e "Huomo piacevole, saria buon canevaro" (Montanari \& Pucci 2009: 171). Non a caso, il termine "Huomo" è qui associato ad aggettivi che valorizzano le qualità umane dell'oste: l'aggettivo "amorevole" evoca l'idea dell'attenzione nei confronti degli altri e l'essere servizievole. L'aggettivo "piacevole" richiama la dimensione conviviale che contraddistingue la taverna, dove l'oste stesso discorre e interagisce con gli avventori, favorendo in tal modo anche il rapporto fra i clienti medesimi. La canova o anche canoba, come si legge nei documenti d'archivio tardo-medievali, poteva essere la cantina o magazzino della locanda in cui l'oste teneva il vino, ma spesso corrispondeva anche al luogo stesso di consumazione delle bevande. Che i vocaboli impiegati per designare i mestieri dell'ospitalità fossero percepiti similari, si rileva dall'impiego indifferentemente dell'uno o dell'altro, come si evince dal semplice "La va da hoste a taverniero" (Montanari \& Pucci 2009: 173). In lingua italiana l'oste è proprietario o gestore di un'osteria, di una locanda; può essere anche un vinattiere, un vinaio, un locandiere. In un'accezione più antica l'oste è sia colui che richiede ospitalità, che chi ne usufruisce ${ }^{15}$. L'osteria (o ostaria), a sua volta, è una locanda provvista di stallatico, sorta per lo più lungo le strade di comunicazione in cui gli avventori possono trovare vitto e alloggio ${ }^{16}$. La taverna (o taberna) è un'osteria per lo più d'infimo ordine e frequentata da persone scioperate $\mathrm{e}$ da ubriaconi; denominata anche bettola ${ }^{17}$. Il tavernaio è il proprietario di una taverna; declinato al femminile tavernaia assume spesso una connotazione negativa, di donna dissoluta e viziosa ${ }^{18}$. Il termine albergo indica un luogo dove si alloggia a pagamento, un edificio destinato ad accogliere i forestieri, soprattutto per dormire ${ }^{19}$. L'albergatore è colui che dà albergo; ma anche

\footnotetext{
${ }^{15}$ GDLI (XII: 242).

${ }^{16}$ GDLI (XII : 251).

${ }^{17}$ GDLI (XX: 769).

${ }^{18}$ GDLI (XX: 769).

${ }^{19}$ GDLI (I: 288-289).
} 
chi offre ospitalità gratuita ${ }^{20}$. La locanda è una sorta di albergo economico di categoria assai modesta ${ }^{21}$.

La letteratura proverbiale di età moderna eredita da quella medievale l'immagine dell'oste quale individuo furbo e scaltro, mosso unicamente dai propri interessi economici, piuttosto che da ideali di accoglienza e di ospitalità, come sembra evocare il proverbio registrato da Croce "Chi fa conto senza l'oste, la fa due volte" (Montanari \& Pucci 2009: 168). Si tratta anche in questo caso di un detto che ha avuto grande fortuna nel tempo, tanto da essere vivo ancora oggi e utilizzato nel linguaggio parlato. Mediante la diffusa ricezione di proverbi del genere, la furbizia, la scaltrezza e l'avidità sono divenute per l'appunto caratteristiche proverbiali dell'oste. L'idea di contabilità, inoltre, è di frequente sottointesa laddove si evocano il guadagno e l'interesse economico, conseguiti, sembra suggerire la maggior parte dei proverbi, in maniera non corretta e a scapito degli avventori. E un mondo, infatti, quello dell'ospitalità rappresentato nei proverbi, fortemente caratterizzato da un'impronta mercantile, proprio perché l'osteria, l'albergo e la taverna potevano trasformarsi durante il giorno in micro-mercati coperti di beni alimentari. Del pari, l'oste, l'albergatore e il taverniere diventavano dei veri e propri mercanti che acquistavano sul mercato cittadino i beni alimentari necessari e li rivendevano nella propria locanda. Ritorna anche nel repertorio proverbiale di Croce la formula assai diffusa nella letteratura tardo-medievale e del primo Rinascimento dell'enumerazione di elementi giustapposti, in una sorta di crescendo narrativo. Il proverbio risulta inserito in quel gusto linguistico per il numero e per la forza simbolica e quasi magica che da esso sprigionava, secondo la cultura del tempo. A questo si aggiunge la dimensione giocosa di tipo linguistico che caratterizza tale genere di formule. È il caso evidente del tre, il numero perfetto per eccellenza, che ricorre spesso, come per esempio il detto riportato da Croce "Tre parti vogliono avere gli osti, cioè buon vino, buon letto e buone chiacchere" (Montanari \& Pucci 2009: 175). Il proverbio appena citato evoca tre qualità fondamentali del buon oste quasi sotto forma di slogan pubblicitario, ovvero fornire un servizio di qualità riguardo al vitto (con particolare riferimento al vino) e all'alloggio; e offrire ai propri avventori un ambiente di convivialità e socievolezza, come l'espressione "buone chiacchere" suggerisce. Affiora, infine, il tema del vino quale simbolo della convivialità e dell'ospitalità tout court, secondo una tradizione proverbiale che trova riscontri e attestazioni sin dall'antichità. Il vino costituisce un simbolo universale di idee, di riferimenti e valori, che molto si presta a rappresentare sia gli aspetti positivi che quelli negativi dell'ospitalità a pagamento. Quest'ultima viene evocata

\footnotetext{
${ }^{20}$ GDLI (I: 288).

${ }^{21}$ GDLI (IX: 178).
} 
da immagini ricorrenti, come l'oste furbo attaccato al suo interesse e la meretrice intenta a fare i propri affari. L'oste, però, può ricevere giudizi positivi se pensa al benessere della clientela. Sembra persistere, tuttavia, nel corso dei secoli, l'idea di taverniere legata al guadagno e alla truffa a danno altrui, come attestano vari modi di dire odierni: "contare senza l'oste" (fare progetti, prendere decisioni senza valutare le eventuali difficoltà); "dare fede all'oste (dare un'apparenza di maggiore credibilità a ciò che si dice); "fare conto d'oste" (truffare sul conto, cercando di farsi pagare più del dovuto); "uccellarsi 1'oste" (farsi beffe, prendere in giro un altro) 22 .

\section{BIBLIOGRAFIA}

Angiolieri, C. (1990). Le rime. Roma: Archivio Guido Izzi.

Balletto, L. (2010). Tavernieri dell'estrema Liguria di Levante a Genova intorno alla metà del Trecento. Giornate di Studio in memoria di Ferdinando Carrozzi, Memorie della Accademia Lunigianese di Scienze Giovanni Capellini, 80, 55-99.

Balletto, L. (2014). Il mondo del vino a Genova intorno alla metà del Trecento. In A. Carassale \& L. Lo Basso (a cura di), In terra vineata. La vite e il vino in Liguria e nelle Alpi marittime dal medioevo ai nostri giorni. Studi in memoria di G. Rebora (pp. 150-204). Ventimiglia: Philobiblon Edizioni.

Battaglia, S. (1961). Grande dizionario della lingua italiana, I. Torino: UTET.

Battaglia, S. (1975). Grande dizionario della lingua italiana, IX. Torino: UTET.

Battaglia, S. (1984). Grande dizionario della lingua italiana, XII. Torino: UTET.

Battaglia, S. (2000). Grande dizionario della lingua italiana, XX, Torino: UTET.

Bautier, A. M. (1984). Peuples, provinces et villes dans la littérature proverbiale latine du Moyen Âge. In F. Suard \& C. Buridant (éd.), Richesse du proverbe, I. Le proverbe au Moyen Âge (pp. 1-22). Lille: Université de Lille III.

Brambilla Ageno, F. (1984). I “proverbi di ser Garzo”. Studi petrarcheschi, n.s., $1,1-37$.

Buridant, C. (1984). Les proverbes et la prédication au Moyen Âge. De l'utilisation des proverbes vulgaires dans les sermons. In F. Suard \& C. Buridant (éd.), Richesse du proverbe, I. Le proverbe au Moyen Âge (pp. 23-45). Lille: Université de Lille III.

Cherubini, G. (1996). L'Italia rurale del basso Medioevo, Roma-Bari: Laterza.

${ }^{22}$ GDLI (XII: 241-242). 
Cherubini, G. (1997). Il lavoro, la taverna, la strada. Scorci di Medioevo. Napoli: Liguori.

Contini, G. (a cura di) (1960). Poeti del Duecento, I. Milano-Napoli: Ricciardi.

Freedman, P. (2012). Eating Out. In K. Albala (ed.), A cultural history of food in the Renaissance, X (Vol. 3, pp. 101-115). London-New YorkBerg: Bloomsbury.

Gautier, A. (2012). Eating Out in the Early and High Middle Ages. In M. Montanari (ed.), A cultural history of food in the medieval age (Vol. 2, pp. 91-106). London-New York-Berg: Bloomsbury.

Gensini, S. (a cura di) (2000). Viaggiare nel Medioevo. Roma: Ministero per $\mathrm{i}$ beni e le attività culturali. Ufficio centrale per i beni archivistici.

Giusti, G. (1993). Raccolta di proverbi toscani. Firenze: Le Monnier.

Kümin, B. (2012). Eating Out in Early Modern Europe. In B. Kümin, A cultural history of food in the modern early age (Vol. 4, pp. 87-101). London-New York-Berg: Bloomsbury.

Latini, B. (1985). Il Tesoretto. Milano: Rizzoli.

Mazzi, M. S. (1997). Oltre l'orizzonte. In viaggio nel Medioevo. Torino: Paravia.

Mengaldo, P. V. (2012). Filologia testuale e storia linguistica. In E. Pasquini (a cura di), Studi e problemi di critica testuale: 1960-2010. Per i 150 anni della Commissione per i testi di lingua (pp. 19-35). Bologna: Commissione per i testi di lingua.

Mitelli, G. M. (1988). Costume e società nei giochi a stampa di Giuseppe Maria Mitelli. Perugia: Electa Editori Umbri Associati.

Montanari, M. (2008). Il formaggio con le pere. La storia in un proverbio. Roma-Bari: Laterza.

Montanari, M. \& Pucci, F. (2009). Fra oralità e scrittura. Frammenti di cultura alimentare nei proverbi di Giulio Cesare Croce. In Z. Zanardi (a cura di), Le stagioni di un cantimbanco: vita quotidiana a Bologna nelle opere di Giulio Cesare Croce (pp. 133-175). Bologna: Editrice Compositori.

Novati, F. (1910). Le serie alfabetiche proverbiali e gli alfabeti disposti nella letteratura italiana dei primi tre secoli. Torino.

Paolo da Certaldo. (1945). Libro di buoni costumi. Firenze: Le Monnier.

Peyer, H. C. (1990). Viaggiare nel Medioevo. Dall'ospitalità alla locanda. Roma-Bari: Laterza.

Pucci Donati, F. (2012). Frammenti di cultura alimentare nella tradizione proverbiale italiana dei secoli XIII-XV. Studi medievali, LIII, 1-81.

Rinaldi, R. (2016). Nella rete delle istituzioni. In F. Pucci Donati \& R. Rinaldi, Consumi e ospitalità in una città di mercati dal Duecento al 
Quattrocento (pp. 165-178). In A. Campanini (a cura di), Bologna e il cibo. Percorsi archivistici nel Medioevo della “Grassa”(pp. 163-205). Bra (Cn): Slow Food Editore.

Sacchetti, F. (2004). Il Trecentonovelle. Torino: Unione tipografico-editrice torinese.

Schulze-Busacker, E. (2012). La didactique profane au Moyen Âge. Paris: Classiques Garnier.

Shapin, S. (2001). Proverbial Economies: how an understanding of some linguistic and social features of common sense can throw light on more prestigious bodies of knowledge, science for example. Social Studies of science, 31/5, 731-769.

Tagliabue, M. (2003). Bere in taverna. In G. Archetti (a cura di), La civiltà del vino. Fonti, temi e produzioni vitivinicole dal Medioevo al Novecento. Atti del Convegno (Monticelli Brusati-Antica Fratta 5-6 ottobre 2001) (pp. 599-634). Brescia: Centro culturale artistico di Franciacorta e del Sebino.

Tuliani, M. (1994). Osti, avventori e malandrini. Alberghi, locande e taverne a Siena e nel suo contado tra Trecento e Quattrocento. Siena: Protagon Editori Toscani.

Zemon Davis, N. (1980). Le culture del popolo. Sapere, rituali e resistenze nella Francia del Cinquecento. Torino: Einaudi.

\section{INNKEEPERS AND INNS IN ITALIAN PROVERBS \\ BETWEEN THE MIDDLE AGES AND THE EARLY MODERN AGE}

Sum mary

Commercial hospitality has always been a recurrent theme in vernacular literature. The tavern, inn and hotel are often mentioned in medieval proverbs, just as the innkeeper, tavern keeper and hotelkeeper. These professionals are frequently depicted as symbols of trickery and greed, rarely of honesty and generosity. They are often individuals who commercialise human relationships and deceive others. A woman, in particular, is usually described as a harlot committed to a disorderly life. A man, on the other hand, is not only represented as a merchant selfishly dedicated to his own enrichment; he can also embody the innkeeper who carries out his work honestly. Proverbs related to hospitality, therefore, have an ethical meaning going beyond the inn's world itself. They stigmatise certain behaviour and negative human qualities, but at the same time contribute to creating in the popular imagination the idea of an innkeeper which remains alive even today. In this respect, proverbial culture provides several keys for reading society, beliefs and morals of the past.

Keywords: hospitality, proverbs, Middle Ages, innkeepers, tavern, morality. 